\title{
Measurement of the temporal coherence properties of pulsed single-mode laser diodes
}

\author{
Michael Haney and Demetri Psaltis
}

\begin{abstract}
The coherence of a single-mode laser diode, under high-speed pulsed modulation, is limited by instabilities in the lasing wavelength arising from transient phenomena in the junction region of the laser. This paper reports the results of an experiment to characterize the effects of these modal instabilities on the temporal coherence of pulsed laser diodes. The primary intent of the experiment was measurement of the cumulative effect of the modal instabilities on the fringe visibility in interferometric time integrating optical processors. A conclusion of this study is that commercially available laser diodes can be used as pulsed light sources in interferometric applications in which the pulse width of the laser is long compared to its characteristic coherence time constant and short compared to its characteristic thermal time constant. Furthermore, the interpulse modal instability can be minimized by prudent choice of operating conditions.
\end{abstract}

\section{Introduction}

The advancement of the state of the art of commercially available laser diodes in recent years has led to their wide use in optical communication and information processing systems. ${ }^{1-3}$ Laser diodes are particularly attractive for applications in which volume and power consumption are constrained due to their small size and high efficiency. In addition laser diodes can be modulated directly with bandwidths of up several gigahertz. When laser diodes are pulsed or modulated the spectral purity of the radiation is reduced from that achievable under $\mathrm{cw}$ operation, and the temporal coherence decreases accordingly. This paper reports the results of a study to characterize the phenomena which degrade the coherence of single-mode laser diodes under pulsed operation.

A primary factor affecting the coherence of pulsed laser diodes is the rise in temperature of the junction area during the pulse due to ohmic heating. ${ }^{4}$ This effect causes a change in the lasing wavelength and, therefore, degrades the temporal coherence. Analytical models for this behavior, based on the thermodynamic properties of the laser diode structure, $, 5,6$ are in good agreement with measured results. 7,8 Other phenomena which affect the temporal coherence of pulsed laser

\footnotetext{
The authors are with California Institute of Technology, Department of Electrical Engineering, Pasadena, California 91125.

Received 26 December 1984.

0003-6935/85/131926-07\$02.00/0.

(ㄷ) 1985 Optical Society of America.
}

diodes include the coherence time constant of the laser and mode hopping. In this paper these effects are characterized individually for commercially available single-mode laser diodes, and the overall coherence function resulting from the combination of the effects is modeled and measured.

The coherence time constant of the laser diode is defined as the time after the onset of laser oscillation in which the laser changes from its initial multimode operation to a single dominant lasing mode. The coherence time constant sets a lower bound on the pulse width, since it must be a negligible portion of the pulse width to obtain coherence. After single-mode operation has been established, the stability of the laser mode is affected by junction heating, which leads to a continuous change in the lasing wavelength throughout the duration of the pulse and intrapulse mode hopping which results in an abrupt change in wavelength. When considering time integrating interferometric systems, where many pulses may be integrated, the pulse-topulse or interpulse mode stability must also be determined. If the laser does not consistently select the same mode in which to oscillate for every pulse, the coherence will be degraded. Finally, it should be mentioned that the coherence properties of the laser diode are influenced by the operating conditions, and, therefore, measurements were made over a wide range of operating conditions.

Most of the measurements were made on the Hitachi HLP-1600 laser. Measurements were also made on the Mitsubishi ML-3001 and RCA C86030E lasers for comparison. Unless otherwise stated, the data presented are for the Hitachi device. The experimental setup with which the measurements were made is described in the next section. The experimental results are presented as well as the trade-offs that exist between performance and operating conditions. Finally, the 
coherence function for the pulsed laser diode is derived and compared with the experimental results.

\section{Experiment}

The pulsed laser diode coherence measurement setup is shown schematically in Fig. 1 . The setup is a Michelson interferometer consisting of mirrors $M 1$ and $M 2$ and the beam splitter $B 1$. Mirror $M 1$ is mounted on a micrometer stage to allow variations in optical path difference (OPD) from 0 to $5 \mathrm{~cm}$. Mirror $M 2$ is mounted on a piezoelectric translator to allow convenient monitoring of the fringe depth at the output of the interferometer. The laser drive electronics consist of a current pulser with variable rise time and dc biasing capability.

The light at the output of the interferometer is detected by a high-speed avalanche photodiode and independently by an integrating linear photodiode array. Beam splitter $B 2$ permits simultaneous data collection with the two types of detector. The avalanche photodiode that was used is capable of measuring rise times of 1 nsec. The photodiode array temporally integrates the interferometrically detected light of many sequential pulses to characterize the pulse-to-pulse coherence of the laser. When data are being taken with the photodiode array the output beams of the interferometer are slightly misaligned along the dimension of the photodiode array by tilting mirror $M 1$. This produces a linear fringe pattern on the array, and the fringe visibility of the interference pattern that is observed on the array is a measure of the coherence over many pulses.

\section{A. Coherence Time Constant}

The coherence time constant is estimated by measuring the time from the beginning of detectable light intensity to the time of maximum fringe visibility. This is accomplished by modulating the OPD with mirror $M 2$ by $\pm \lambda / 2$ at $\sim 1 \mathrm{kHz}$ with the piezoelectric translator. When many individual pulses are simultaneously displayed on the screen of the oscilloscope, the persistence of the screen's phosphor causes the trace to smear due to the varying relative phase between the interfering beams. This smearing occurs only when the laser is oscillating in a single longitudinal mode. During the start-up period of the pulse, the light is incoherent, and thus the trace of many pulses appears as a single line. The coherence time constant is approximately the difference between the time light is first detected as a single trace and the time of maximum smearing due to interference.

Figure 2 shows the interferometric activity at the leading edge of the laser pulse. The first $8 \mathrm{nsec}$ of a 50-nsec pulse with $15-\mathrm{mW}$ peak power are shown. The OPD for the data of Fig. 2 is $1 \mathrm{~cm}$, and the time scale of the displayed data is $1 \mathrm{nsec} / \mathrm{div}$. The envelope of the trace in Fig. 2 is a measure of the modulation depth of the interference, and thus it provides an estimate of the coherence time. The coherence time constant in Fig. 2 is $\sim 1.5$ nsec. Prebiasing the laser is a dc level just below the threshold current of the laser reduces the coherence time constant slightly but not sufficiently to

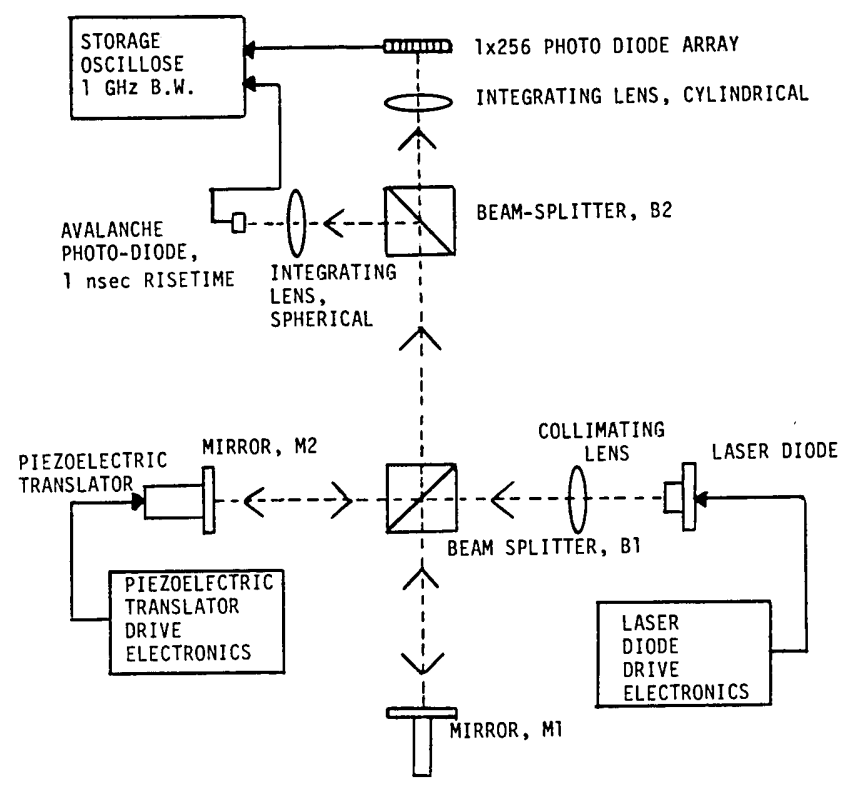

Fig. 1. Laser diode temporal coherence measurement setup.

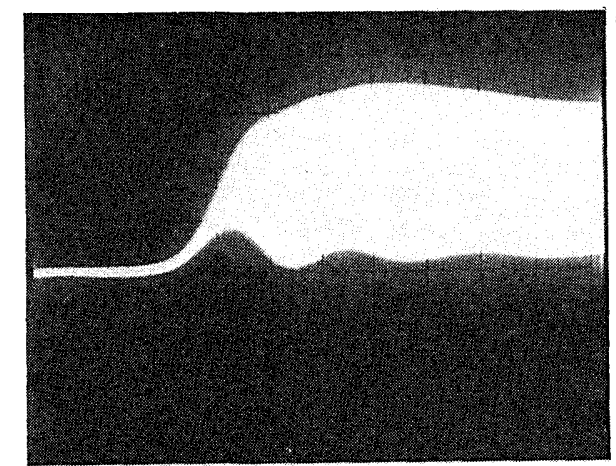

Fig. 2. Coherence time measurement, 1 nsec/div.

allow accurate measurements of the effect at the short (subnanosecond) time scale involved.

The coherence time constants of the Mitsubishi and RCA lasers were also measured. These lasers have coherence time constants comparable with those of the Hitachi laser diode. At large peak power $(>35 \mathrm{~mW})$, however, the coherence time constant for the RCA laser diode became dramatically longer. In this case, the shape of the interferometrically detected light pulse indicated that two or more competing modes continued to oscillate simultaneously for periods of up to $50 \mathrm{nsec}$ before one became dominant.

In conclusion, it was found that the coherence time constant of the single-mode lasers we tested was between 1 and $2 \mathrm{nsec}$. Therefore, if the laser pulse is much longer than $2 \mathrm{nsec}$, the coherence time constant will not significantly affect the coherence properties of the laser. For example, if 50-nsec pulses are used, the laser will be oscillating in a single mode $96-98 \%$ of the time.

\section{B. Intrapulse Stability}

The intrapulse stability of the lasing mode is measured by studying the trace of the output of the ava- 


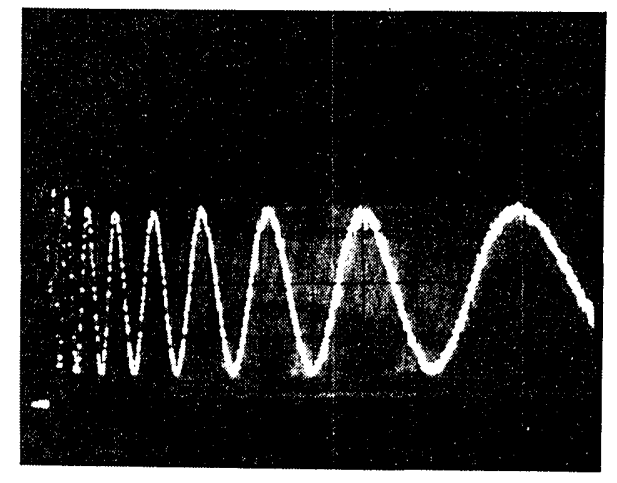

(a)

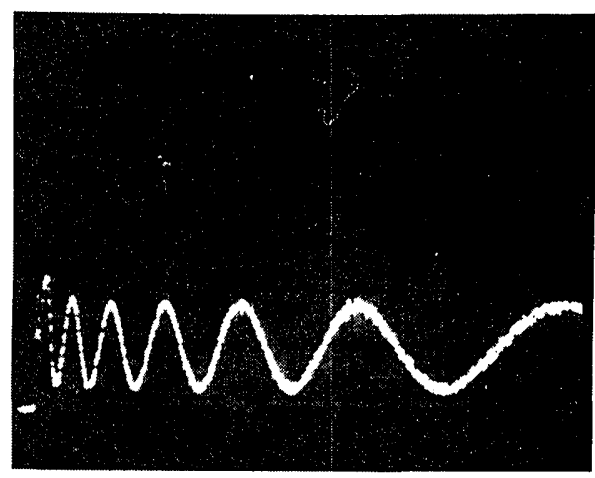

(b)

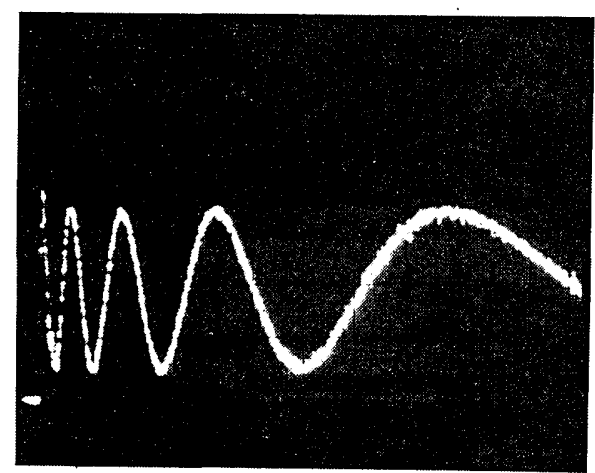

(c)

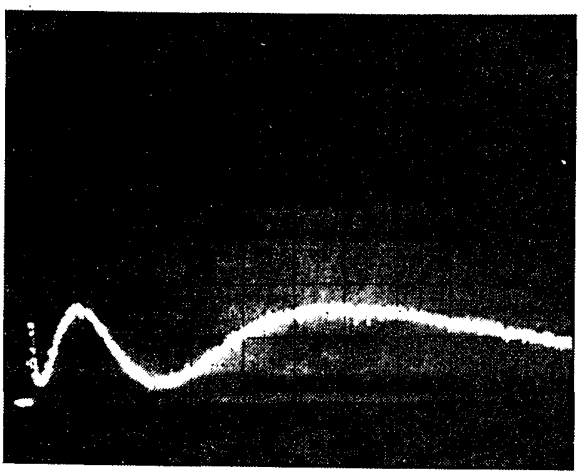

(d)
Fig. 3. Modal instability induced by junction heating during the pulse; (a) peak power $=15 \mathrm{~mW}$, bias $=0,200 \mathrm{nsec} / \mathrm{div}$; (b) peak power $=8 \mathrm{~mW}$, bias $=0,200$ nsec/div; (c) peak power $=15 \mathrm{~mW}$, bias $=50 \mathrm{~mA}, 200 \mathrm{nsec} / \mathrm{div}$; (d) peak power $=8 \mathrm{~mW}$, bias $=50 \mathrm{~mA}$, $200 \mathrm{nsec} / \mathrm{div}$. lanche photodiode for a single pulse of light from the interferometer with mirror $M 2$ kept stationary. Changes in the lasing wavelength during the pulse are monitored as intensity variations of the interferometrically detected waveform.

Two distinct types of intrapulse instability were observed. The first is attributed to the junction heating during the pulse causing a continuous shift in the lasing wavelength with time. The second phenomenon affecting the intrapulse stability is mode hopping in which the laser changes between two adjacent longitudinal modes during the pulse. Both phenomena were observed and characterized.

The effect of a continuous shift in the lasing wavelength on the interferometrically detected signal is shown in Fig. 3. Figure 3 shows oscilloscope traces of the output of the avalanche photodiode for individual laser pulses under four different sets of operating conditions. The duration of the pulses in Fig. 3 is $2 \mu$ sec. The OPD for these measurements was $4 \mathrm{~cm}$. The pulse width and OPD were chosen so that the interferometric effects could be observed over a wide range of operating conditions. It is noted that the frequency of the intensity modulation is highest in the early part of the pulse and seems to asymptotically go to zero.

The effects of operating conditions on the mode stability are evident in Fig. 3. Figures 3(a) and (b) show the interferometer output for laser peak output power of 15 and $8 \mathrm{~mW}$, respectively, without dc biasing. The smaller number of cycles in Fig. 3(b) indicates a lower rate of change of temperature. Figures $3(c)($ and $(d)$ show the effect of prebiasing the laser at its threshold current level for 15 and $8-\mathrm{mW}$ peak laser intensity, respectively. The significant reduction in the number of cycles observed is attributed to the fact that prebiasing raises the temperature of the laser, and the subsequent change in temperature during the pulse is reduced.

To explain the observations we must first understand how the laser wavelength changes with junction temperature. The wavelength of a single-longitudinalmode laser is given by the following 9 :

$$
\lambda=2 n L / q,
$$

where $\lambda$ is the wavelength, $L$ is the length of the cavity, $n$ is the index of refraction, and $q$ is an integer. The change in wavelength with temperature is then given by

$$
\lambda^{\prime}=d \lambda / d T=2 / q(n d L / d T+L d n / d T) .
$$

For small variations about room temperature, $\lambda^{\prime}$ is approximately constant. The HLP-1600 has a measured $\lambda^{\prime}$ of $0.06 \mathrm{~nm} / \mathrm{K} .{ }^{10}$

The detected intensity due to the interference of the two beams is proportional to the cosine of the phase difference $\Delta \beta$ of the waves:

$$
\Delta \beta=2 \pi d / \lambda
$$

where $d$ is the OPD. The change in phase difference with changing $\lambda$ is then given by

$$
d(\Delta \beta) / d \lambda=-2 \pi d / \lambda^{2} .
$$

We multiply Eq. (4) by $\lambda^{\prime}$ to determine the effect of the 
temperature change on the phase difference measured with the interferometer:

$$
d(\Delta \beta) / d T \simeq-2 \pi d \lambda^{\prime} / \lambda^{2}
$$

The change in junction temperature during the beginning of laser oscillation has been modeled as follows 5 :

$$
\Delta T(t)=T_{0}[1-\exp (-t / \tau)],
$$

where $T_{0}$ is the asymptotic value of the junction temperature change, and $\tau$ is the thermal time constant which is determined by the thermal resistance and capacitance of the junction. By integrating Eq. (6) and substituting in Eq. (7) the following expression for the phase difference as a function of time is obtained:

$$
\Delta \beta(t)=2 \pi d T_{0} \lambda^{\prime}[1-\exp (-t / \tau)] / \lambda^{2} .
$$

The change in junction temperature is plotted in Fig. 4 as a function of time from the data in Fig. 3. The phase of the amplitude modulation is used as a measure of the change in junction temperature with time. The data clearly show that the temperature rise is exponential in nature and is a close match to the model of Eq. (7) with time constants that are equal to several hundred nanoseconds.

The rates of wavelength shift of the RCA and Hitachi lasers were compared at an output power of $20 \mathrm{~mW}$ for 100 -nsec pulse widths. It was found that the wavelength of the RCA laser shifted at approximately one half of the rate of the Hitachi laser. This is attributed to the larger optical cavity of the RCA device which leads to a longer thermal time constant.

Mode hopping is the second type of intrapulse mode instability that was observed. This phenomenon manifests itself as a discontinuity in the intensity measured at the output of the interferometer. Figure 5 illustrates the effect of an intrapulse mode hop in a 20-mW pulse. The OPD, pulse amplitude, and pulse width were chosen sufficiently large that the effect of the junction heating as well as the intrapulse mode hopping were observed. The pulse width in Fig. 5 is 800 nsec, and the OPD is $4 \mathrm{~cm}$. At this particular OPD the sudden change in mode during the pulse manifests itself as a step change in the phase of the intensity modulation of $\sim 180^{\circ}$ indicating that the OPD is close to a value which is an integral number of $\lambda^{2} / 2(\delta \lambda)$, where $\delta \lambda$ is the intermode spacing of the laser.

In general, the probability of occurrence of an intrapulse mode hop increases at higher pulse amplitude and longer pulse widths. For the Hitachi laser diode it was found that for a pulse width of $50 \mathrm{nsec}$, the frequency of intrapulse mode hops is $<1 / 40$ for a peak power level of $12 \mathrm{~mW}$ or less. The RCA laser at $12 \mathrm{~mW}$ was found to have about twice the rate of mode hopping of the Hitachi laser.

The rates of mode hopping given above are averaged. The actual mode hopping rates varied significantly from measurement to measurement. Intrapulse mode hopping occurs because the positions of the modes shift in relation to the gain curve of the laser as the temperature of the junction region rises during the pulse. If

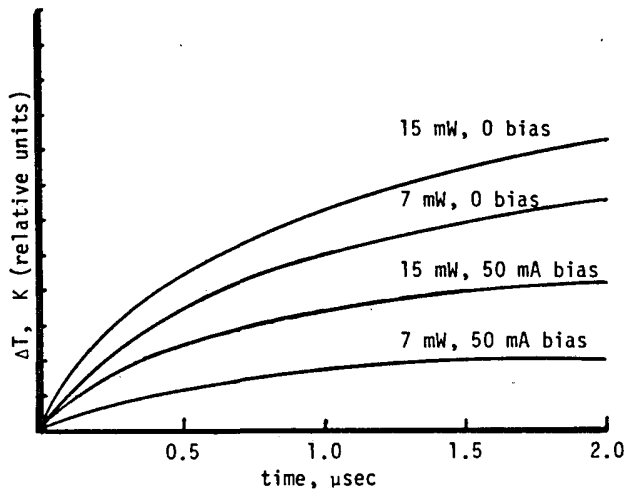

Fig. 4. Rise in junction temperature for different operating conditions.

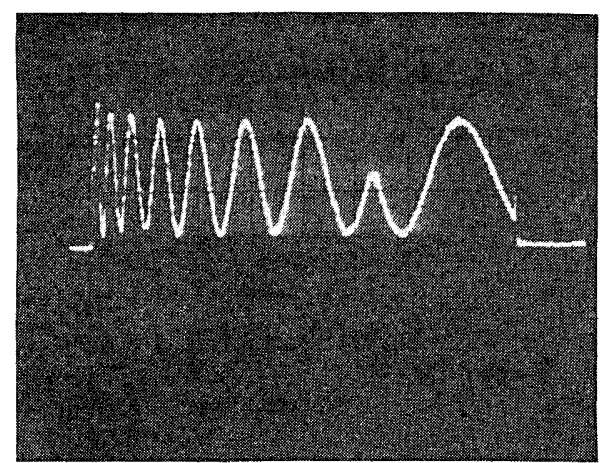

Fig. 5. Intrapulse mode hop, peak power $=20 \mathrm{~mW}$, bias $=0,100$ nsec/div.

the shift is large enough, an adjacent mode will move closer to the center of the gain curve than the current lasing mode and will take over. Slight changes in the ambient temperature of the laser's heat sink will also manifest themselves as shifts in the mode position relative to the laser's gain curve thereby changing the mode hopping rate and/or the position within the pulse at which the mode hop occurs.

\section{Interpulse Stability}

Interpulse instabilities occur when over a long sequence of pulses the laser does not always choose the same mode in which to oscillate. This effect was also characterized in the experimental setup of Fig. 1 . The pulse repetition frequency was set at $20 \mathrm{kHz}$, the pulse width at $50 \mathrm{nsec}$, and the peak power at $10 \mathrm{~mW}$. The OPD for these data was $1 \mathrm{~mm}$. Under these operating conditions intrapulse mode hopping is negligible. Furthermore, the change in the phase difference between the interfering beams due to junction heating is small enough so that the resulting output can be considered a sampled version of the $\mathrm{cw}$ case. The OPD was modulated by $\pm \lambda / 2$ at a frequency of $1 \mathrm{kHz}$. Modulating the OPD in this manner reveals the fringe depth of the interfering beams. An interpulse mode hop will manifest itself as a discontinuity in the sampled waveform. The output of the avalanche photodiode was monitored on the oscilloscope, and an oscilloscope trace of this measurement is shown in Fig. 6. Figure 6 shows 
that one mode is dominant, but occasionally an adjacent mode is selected. Different modes will, in general, have different relative phases between the output beams of the interferometer. This causes the detected intensities for the two modes to be different, as shown in Fig. 6. It is clear that for time integrating interferometric systems, in which the light from many laser pulses is detected interferometrically and summed, interpulse mode hopping could severely degrade the fringe visibility and thus must be minimized.

As with the intrapulse mode hopping, the rate of interpulse mode hops was found to be determined by the electrical operating conditions of the laser. In general, the laser was more stable (a single mode was more likely to dominate) at lower peak power levels. This is probably due to the more gradual buildup of laser oscillation at the lower drive current levels resulting in more consistent selection of the dominant mode. It was also found that the rate of mode hopping could be significantly reduced by altering the shape of the current drive pulse to have a slower rise time. Measurements of the interpulse mode hopping rates were made for different drive pulse shapes and peak power levels. For these measurements the slope of the leading edge of the drive pulses was varied while maintaining the peak level pulse width at $\sim 50$ nsec. Figure 7 shows oscilloscope traces showing the shapes of typical drive pulses that were compared. For the Hitachi laser it was found that for 10-nsec rise times, the interpulse mode stability was $>95 \%$ for peak power levels below $6 \mathrm{~mW}$. However, for drive pulses with rise times of $80 \mathrm{nsec}$, such as in Fig. $7(\mathrm{~b})$, the laser diode exhibited $>95 \%$ stability for peak power levels up to $12 \mathrm{~mW}$. For rise times $>80$ nsec no measurable improvement in the interpulse mode stability was observed. Furthermore, it was noted that applying a dc bias current to the laser at a level below the threshold current did not significantly affect interpulse stability.

\section{Coherence Function Measurement}

The four types of instability phenomenon that were discussed in the previous sections combine to degrade the fringe visibility in an interferometric system. The relative importance of these instabilities to coherence degradation depends on the operating conditions of the laser diode and the OPD of the optical system. The effect of the coherence time constant is minimal if it is short when compared to the pulse width. For the measurements reported here this condition was generally met, and, therefore, the coherence time constant was a minor factor. Furthermore, we found that intrapulse and interpulse mode hopping are minimized by proper choice of operating conditions.

For example, with the Hitachi laser at a peak power of $10 \mathrm{~mW}$, the combined intrapulse and interpulse mode hopping occurs at a rate of less than 1 mode hop in twenty pulses for a current drive pulse whose shape is similar to that of Fig. 7(b). The most significant instability is the wavelength shift due to the heating of the junction area during the pulse. To maintain a high degree of coherence for a particular OPD, the pulse

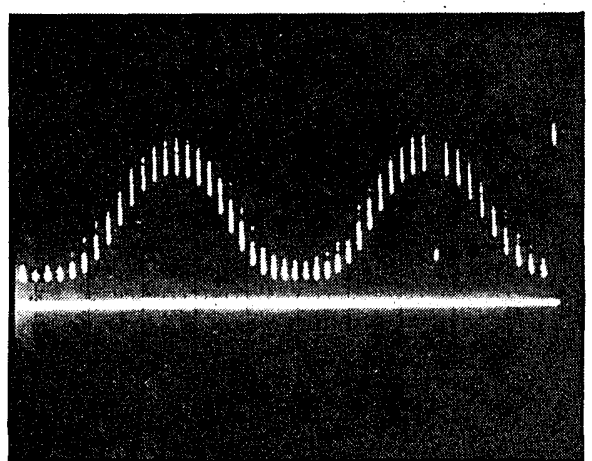

Fig. 6. Interpulse mode hopping.

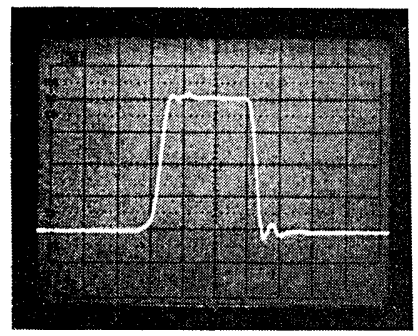

(a)

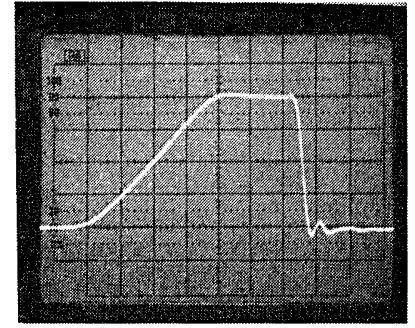

(b)
Fig. 7. Laser drive pulses: (a) 10-nsec rise time; (b) 80-nsec rise time.

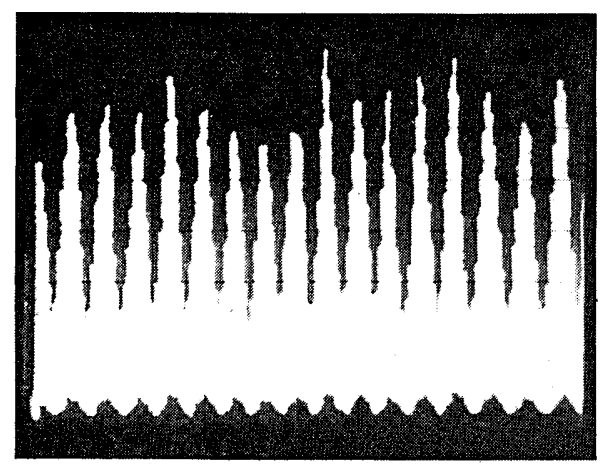

Fig. 8. Fringe pattern generated by 10,000 interferometrically detected $\mathrm{LD}$ pulses.

width must be such that the intensity measured at the output of the interferometer will fluctuate only a fraction of one of the cycles shown in the examples of Fig. 3.

Evaluation of the combined effects of the individual instabilities on the visibility of the interference pattern that results from the integration of many sequential pulses was accomplished by studying the output of the photodiode array. Figure 8 is an oscilloscope trace of the array output. The trace represents the time integrated interferometric response of $\sim 10,000$ individual laser pulses over a period of $50 \mathrm{msec}$. The data shown were taken at approximately zero OPD and thus represent the largest attainable fringe depth. In the example of Fig. 8 the pulse width is 50 nsec. The actual fringe depth achieved is not $100 \%$ because of several imperfections of the system. The photodiode array has dark noise and signal spreading which will lead to bias 
contributions. Furthermore, the very short multimode portion at the beginning of each pulse adds incoherently to result in a contribution to the bias.

A measurement of the fringe visibility, normalized to the maximum fringe visibility obtained at $\mathrm{OPD}=0$, as in Fig. 8, provides a direct measurement of the coherence function under different operating conditions. The measured fringe visibility as a function of OPD for different pulse widths is plotted in Fig. 9. In these data the effects of mode hopping have been minimized, leaving junction heating as the primary factor that is responsible for reduced fringe visibility.

\section{Coherence Function Analysis}

In this section we derive the coherence function for pulsed laser diodes based on the model of the thermodynamic properties of the laser junction given in Eq. (6). The coherence function analysis is then extended to time integrating systems by including the effect of interpulse mode hopping.

The coherence time constant of a single-mode laser diode has been treated theoretically in the literature.11 It was predicted that, at moderate to high peak power levels, one longitudinal mode dominates very rapidly $(\sim 1 \mathrm{nsec})$ after the onset of laser oscillation. This is in good agreement with our measurements. The coherence time phenomenon made a negligible contribution to the coherence degradation when the pulse width is very long compared to the coherence time constant. We assume this to be the case and neglect this effect in our analysis. We also ignore intrapulse mode hopping because it was also negligible under the operating conditions used. Therefore, the dominant phenomena degrading the coherence of the laser are the intrapulse modal instability due to junction heating and interpulse mode hopping.

For a single pulse, the coherence function of the single-mode laser diode is assumed to be determined solely by the junction heating as modeled by Eq. (6). This effect is deterministic, and, therefore, in this case the coherence function does not involve ensemble averages. The coherence function for multiple pulses, on the other hand, with interpulse mode hopping, in stochastic in nature. We will first derive an expression for the effects of junction heating and then add the effects of random interpulse mode hopping.

The electric field at the output of the interferometer is given by

$$
E=\exp [j \omega(t) t]+\exp [j \omega(t-d / c)(t-d / c)],
$$

where $d$ is the OPD of the interferometer and $\omega(t)$ is the laser's oscillating frequency which changes with time due to the junction heating effect. From (7) the wavelength as a function of time is given by

$$
\lambda(t)=\lambda_{i}+T_{0} \lambda^{\prime}[1-\exp (-t / \tau)],
$$

where $\lambda_{i}$ is the lasing wavelength at the start of the pulse. Since the change in wavelength due to the junction heating is small compared with $\lambda_{i}$, we have

$$
\omega(t)=2 \pi c / \lambda(t) \simeq\left(2 \pi c / \lambda_{i}\right)\left\{1-\lambda^{\prime} T_{0}[1-\exp (-t / \tau)] / \lambda_{i}\right\}
$$

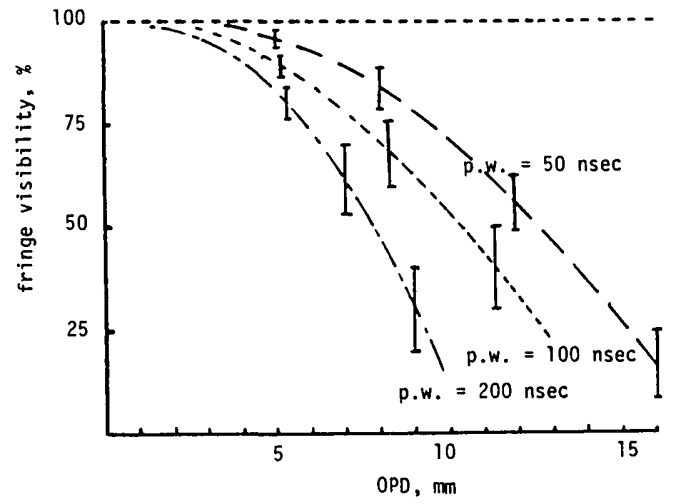

Fig. 9. Measurements of the modulus of the coherence function for 10,000 time integrated pulses.

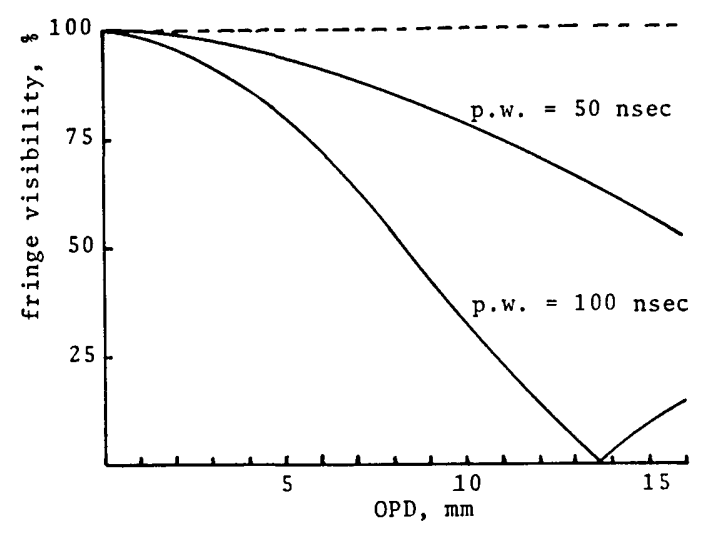

Fig. 10. Theoretical modulus of the coherence function.

We now write an expression for the normalized coherence function as a function of the OPD:

$$
G(d) \simeq\langle\exp [j \omega(t) t] \exp \{-j[\omega(t)(t-d / c)]\}\rangle,
$$

where \langle\rangle indicates time average over the pulse duration $P$. In (11) $\omega(t-d / c)$ is approximated by $\omega(t)$ because $-d / c$ is negligible with respect to $\tau$. We have

$$
G(d)=1 / P \int_{0}^{P} \exp \{j[\omega(t) d / c]\} d t .
$$

Substituting in Eq. (10) and using the assumption that $P \ll \tau$, so that $\exp (-t / \tau) \simeq 1-t / \tau$ on the interval $P$, the integration in Eq. (12) can be performed in closed form:

$$
G(d)=\operatorname{sinc}\left(d c_{1} P\right) \exp \left[j 2 \pi\left(d / \lambda_{i}-d c_{1} P / 2\right)\right],
$$

where $c_{1}=\lambda^{\prime} T_{0} / \lambda_{i}^{2} \tau$ and $\operatorname{sinc}(x)=\sin (\pi x) / \pi x$.

The fringe visibility $V$ is defined as the magnitude of the coherence function. Hence, for pulse widths much less than the thermal time constant, we have

$$
V=\left|\operatorname{sinc}\left(d c_{1} P\right)\right| .
$$

Equation (14) is plotted in Fig. 10 for pulse widths of 50 and 100 nsec. A thermal time constant of $350 \mathrm{nsec}$ is assumed. The measured fringe visibility (Fig. 9) is in good agreement with Fig. 10 . Note that the effect of a large pulse amplitude on the coherence function would 


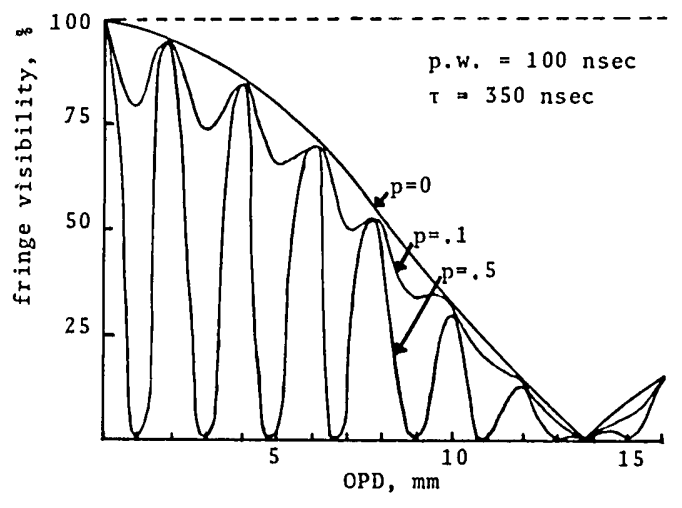

Fig. 11. Calculated effect of interpulse mode hopping on the modulus of the coherence function.

be to increase $T_{0}$ and hence $c_{1}$ in Eq. (14), thus leading to lower coherence.

We now analyze the fringe visibility for the case of two competing adjacent longitudinal modes. The two modes have frequencies $\omega_{1}(t)$ and $\omega_{2}(t)=\omega_{1}(t)+\delta \omega$, where $\delta \omega$ is the Fabry-Perot mode spacing of the laser diode. The time dependence is assumed to be due solely to the junction heating effect. We assign $p$ and $1-p$ to be the probabilities of occurrence of the two modes. For any one pulse, only one of the two modes exists, and thus the coherence function for multiple time integrated pulses is

$$
G_{2}(d)=E(\langle\exp \{j \omega(t) t\} \exp \{-j[\omega(t)(t-d / c)]\})) .
$$

Using Eq. (13) we find

$$
\begin{aligned}
G_{2}(d)= & \exp \left[j\left(\omega_{1, i} d / c-\pi d c_{1} P\right)\right] \operatorname{sinc}\left(d c_{1} P\right) \\
& \times\{p+(1-p) \exp [j(\delta \omega) d / c]\},
\end{aligned}
$$

where we have ignored the insignificant differences in the phase term and in the argument of the sinc function for the two modes. $\omega_{1, i}$ and $\omega_{2, i}=\omega_{1, i}+\delta \omega$ are the initial lasing frequencies for two modes. Hence the fringe visibility is given by
$V_{2}=\left|\sin c\left(d c_{1} P\right)\right|\left\{2 p^{2}-2 p+1+2 p(1-p) \cos [(\delta \omega) d / c]\right\}^{1 / 2}$.

This function is plotted in Fig. 11 for $p=0, p=0.1$, and $p=0.5$ for a pulse width of 100 nsec. Figure 11 depicts the degradation in coherence due to the interpulse mode hopping instability.

\section{References}

1. D. Botez and G. Herskowitz, "Components for Optical Communications Systems: A Review," Proc. IEEE 68, No. 6 (June 1980).

2. J. G Duthie, J. Upatnieks, C. R. Christensen, and R. D. McKenzie, Jr., "Real Time Optical Correlation with Solid State Sources," Proc. Soc. Photo-Opt. Instrum. Eng. 231, 281 (1980).

3. D. Psaltis, "Optical Image Correlation Using Acoustooptic and Charge-Coupled Devices," Appl. Opt., 21, 491, (1982).

4. Y. A. Bykovskii et al., "Method for Investigating Thermal Condition in and Spectral Characteristics of a Semiconductor Laser by Means of a Fabry-Perot Resonator," Sov. Phys. Semicond., 5, 435 (1971).

5. J. Butler, "The Effect of Junction Heating on Laser Linearity and Harmonic Distortion," in Topics in Applied Physics, Vol. 34 (Springer-Verlag, Berlin, 1980), Chap. 8.

6. Y. A. Bykovskii et al., "Coherence of the Radiation of a Pulsed Single Mode Injection Semiconductor Laser," Sov. Phys. Dokl., 17, 359 (1972).

7. P. Melman and W. J. Calsen, "Interferometric Measurement of Thermal Coherence and Time-Varying Longitudinal-Mode Wavelengths in GaAs Diode Lasers," in Technical Digest, Conference on Lasers and Electro-optics (Optical Society of America, Washington D. C., 1981), paper WS4.

8. M. Haney and D. Psaltis, "Coherence Properties of Pulsed Laser Diodes," Proc. Soc. Photo-Opt. Instrum. Eng. 422, 197 (1983).

9. A Yariv, Quantum Electronics (Wiley, New York, 1975).

10. Hitachi Laser Diode Application Manual.

11. K. Y. Lau, C. Harder, and A. Yariv, "Longitudinal Mode Spectrum of Semiconductor Lasers Under High-speed Modulation" IEEE J. Quantum Electron. QE-20, No. 1, 71 (Jan. 1984).

The research reported in this paper is supported by the Air Force Office of Scientific Research, General Dynamics, and the Army Research Office. 